\title{
CHECKLIST OF TEREBRANTIA (THYSANOPTERA: INSECTA) OF DELHI
}

\author{
Vikas Kumar ${ }^{1}$, Kaomud Tyagi ${ }^{2}$ and J.S. Bhatti ${ }^{3}$ \\ ${ }^{1}$ Division of Entomology and Nematology, Indian Institute of Horticultural Research, Hesarghatta, Salt Lake, Bangalore, Karnataka, India \\ ${ }^{2}$ Insect Systematics Laboratory, Project Directorate of Biological Control, Bangalore, Karnataka, India \\ ${ }^{3}$ Department of Zoology, University of Delhi, Delhi, India \\ Email: ${ }^{1}$ vikasnirwal@yahoo.com
}

\begin{abstract}
Eighty-six named species of the suborder Terebrantia (Thysanoptera), in 51 genera and three families are listed along with their synonymies found in Delhi with many species still waiting description. The family-wise breakup being Aeolothripidae with three species in two genera, Stenurothripidae with a single species and Thripidae with 82 species in 48 genera. Eight species are being reported as new to Delhi.
\end{abstract}

\section{KeYwords}

Checklist, Delhi, host plant, new record, Terebrantia, Thysanoptera

Ramakrishna Ayyar (1940) was, probably, the first to start taxonomy of these little insects in India. He along with $\mathrm{V}$. Margabandhu recorded 232 species of Thysanoptera from India in their catalogue which was published in 1940. Professor T.N. Ananthakrishnan is the most important worker who actually gave speed to the taxonomy of Indian Thysanoptera. Ananthakrishnan and Sen (1980) provided keys to the 647 (260 in Terebrantia and 387 in Tubulifera) species from India. Bhatti (1990) in his catalogue of insects of Terebrantia from the Indian subcontinent recorded 290 species in 124 genera in five families.

The first record of Thysanoptera from Delhi was by Singh (1942) who reported Aeolothrips fasciatus, a Terebrantian, from Delhi. His specimens probably represent Coleothrips collaris, a species that was subsequently definitively identified from Delhi (Bhatti, 1964).

A list of the Thysanoptera of Delhi was first provided by Bhatti (1997), which include 90 species of Thysanoptera (70 species in 49 genera of Terebrantia and 20 species in 18 genera of Tubulifera).

Bhatti (1997) overlooked two species, which were reported from Delhi before 1997. These species are Dichromothrips corbetti (Priesner, 1936) reported by Mound (1976) on orchid imported from Thailand and Thrips speratus Strassen, 1978 reported by Bhatti (1990).

Bhatti et al. (1999) reported Neohydatothrips gracilipes (Hood, 1924) from Delhi and described a new species Thrips chandni from Delhi (Bhatti, 1999). Neohydatothrips samayunkur (Kudô, $1995)$ is recently reported as new to India from six states including Delhi by Bhatti et al. (2003). Kumar et al. (2005) reported three species of Terebrantia as new to India, all the three species were collected from Delhi.

The present study comprises 86 species of Terebrantia in 51 genera belonging to three families: Aeolothripidae, two genera (with 3 species), Stenurothripidae with a single species and Thripidae (48 genera with 82 species).
Eight species are newly recorded from Delhi. These are: Bathrips melanicornis, Brevithrips tumiceps, Holarthothrips indicus, Sorghothrips fuscus, Thrips carthami, Thrips coloratus, Thrips florum, and Thrips garuda.

\section{Host plant relationship}

The pattern of host utilization amongst the species of a particular group of phytophagous insects most probably reflects, in some way, the ecological and evolutionary strategies adopted by that lineage. Some groups have been, more or less, constrained during their evolutionary history and are associated only with particular group of plants, whereas other groups are coupled with a varied array of plants. Thus, it is imperative to know the host plant of a species in order to study its biology and to carry out any other biological experiments. Little is known about host plant relationship of Delhi Terebrantia, many species being known from too few specimens for any serious information to be available on their host plants and seasonality.

Most of the biologists describe a species as it has been found on such-and-such plant or plants without any indication of true host plant. This information is important from the point of where a species lives, but does not provide insight into its breeding preferences. Further, it has been noticed in most of the publications that such-and-such species is polyphagous without any information regarding the host plant in different seasons and different geographical areas.

Members of genus Scolothrips and Coleothrips are known as obligate predators and facultative predators, respectively. All the three species of Scolothrips from India are found in Delhi, but nothing is known about their host plants except that they are collected in very few numbers from members of family Poaceae. Whether these species of Scolothrips breed in grasses or they have been transported readily by winds is not clear. Coleothrips is represented by two species in Delhi (Coleothrips collaris \& C. mongolicus) out of six in India. Coleothrips mongolicus (Pelikán, 1985) has been found breeding in the flowers of Matthiola incana (Cruciferae) as we have collected adults and immatures for two years but host plant of Coleothrips collaris is still not clear.

Frankliniella schultzei, which is one of the species that are vectors of Tospoviruses in India and worldwide, and has also been reported to be pollinator of chillies and pigeon pea, has been collected from 23 different plants in Delhi. Unfortunately, the actual host where it breeds it still not known from Delhi as well as from India.

Manuscript 1536; (C) ZOO; Date of publication 21 May 2007 Received 10 March 2006; Revised received 25 November 2006; Finally accepted 20 April 2007 


\section{Checklist}

This checklist is the result of investigations on material collected in field studies over the past six years as well as study of material already available from collections made in Delhi during the past four decades. Specimens of all of these species have been studied, and many of the species have been freshly collected during the present investigation.

\section{Family: Aeolothripidae Uzel, 1895}

I.a. Genus Coleothrips Haliday, 1836

1. Coleothrips collaris (Priesner, 1919)

2. Coleothrips mongolicus (Pelikán, 1985)

I. b. Genus Indothrips Bhatti, 1967

1. Indothrips bhushani Bhatti, 1967

II. Family: Stenurothripidae Bagnall, 1923

II. a. Genus Holarthrothrips Bagnall, 1927

1. Holarthrothrips indicus Bhatti \& Anan, 1978

III. Family: Thripidae Stephens, 1829

III. A. Subfamily: Dendrothripinae Priesner, 1925

III.A.a. Genus Dendrothrips Uzel, 1895

1. Dendrothrips cameroni Priesner, 1965

2. Dendrothrips punctatus zur Strassen, 1968

III.A.b. Genus Pseudodendrothrips Schmutz, 1913

1. Pseudodendrothrips albana Bhatti, 1997

2. Pseudodendrothrips suvarna Bhatti,1997

III.B. Subfamily: Panchaetothripinae Bagnall, 1912 III.B.a. Genus Astrothrips Karny, 192

1. Astrothrips stannardi Bhatti, 1967

III.B.b. Genus Brevithrips Bhatti, 1967

1. Brevithrips tumiceps (Karny, 1923)

III.B.c. Genus Caliothrips Daniel, 1904

1. Caliothrips graminicola (Bag. \& Cam., 1932)

2. Caliothrips indicus (Bagnall, 1913)

3. Caliothrips luckmanni Wilson, 1975

III.B.d. Genus Phibalothrips Hood, 1918

1. Phibalothrips peringueyi (Faure, 1925)

III.B.e. Genus Rhipiphorothrips Mor., 1913

1. Rhipiphorothrips cruentatus Hood, 1919

III.C. Subfamily: Sericothripinae Karny, 1921

III.C.a. Genus Hydatothrips Karny, 1913

1. Hydatothrips atactus Bhatti, 1973

2. Hydatothrips ramaswamiahi Priesner, 1926

III.C.b. Genus Neohydatothrips John, 1929

1. Neohydatothrips gracilipes (Hood, 1924)

2. Neohydatothrips raniae (Bhatti, 1967)

3. Neohydatothrips samayunkur (Kudô, 1995)

III.C.c. Genus Pyrothrips Bhatti ,1973

1. Pyrothrips boerhaaviae (Sesh. \& Anan.,1954)

III.D. Subfamily: Thripinae Karny, 1921

III.D.a. Genus Agrostothrips Hood, 1954

1. Agrostothrips meridionalis (Bagnall, 1927)

III.D.b. Genus Ajothrips Bhatti, 1967

1. Ajothrips medius Bhatti 1997

III.D.c. Genus Alathrips Bhatti, 1970

1. Alathrips roonwali (Bhatti, 1963)

III.D.d. Genus Anaphothrips Uzel, 1895

1. Anaphothrips sudanensis Trybom, 1911

III.D.e. Genus Anascirtothrips Bhatti, 1961
1. Anascirtothrips arorai Bhatti, 1967

III.D.f. Genus Ayyaria Karny, 1927

1. Ayyaria chaetophora Karny, 1927

III.D.g. Genus Bathrips Bhatti, 1962

1. Bathrips melanicornis (Shumsher, 1946)

III.D.h. Genus Bolacothrips Uzel, 1895

1. Bolacothrips striatopennatus (Schmutz, 1913)

III.D.i. Genus Bregmatothrips Hood, 1912

1. Bolacothrips brachycephalus (Shumsher, 1942)

III.D.j. Genus Ceratothripoides Bagnall, 1918

1. Ceratothripoides claratris (Shumsher, 1946)

III.D.k. Genus Chirothrips Haliday, 1836

1. Chirothrips africanus Priesner, 1932

III.D.I. Genus Craspedothrips zur Strassen, 1966

1. Craspedothrips minor (Bagnall, 1921)

III.D.m. Genus Dendrothripoides Bagnall, 1923

1. Dendrothripoides innoxius (Karny, 1914)

III.D.n. Genus Diarthrothrips Williams, 1915

1. Diarthrothrips nimbus (Anan., 1965)

III.D.o. Genus Dichromothrips Priesner, 1932

1. Dichromothrips corbetti (Priesner, 1936)

2. Dichromothrips indicus Mound, 1976

III.D.p. Genus Eremiothrips Priesner, 1949

1. Eremiothrips varius (Bhatti, 1967)

III.D.q. Genus Exothrips Priesner, 1939

1. Exothrips cephalicus Bhatti, 1975

2. Exothrips deemax Bhatti, 1975

3. Exothrips hemavarna (Ram. \& Marg., 1931)

4. Exothrips ornus Bhatti, 1975

5. Exothrips poorva Bhatti, 1975

6. Exothrips redox Bhatti, 1975

III.D.r. Genus Florithrips Bhatti, 1970

1. Florithrips traegardhi (Trybom, 1911)

III.D.s. Genus Foliothrips Bhatti, 1972

1. Foliothrips oratus Bhatti, 1972

III.D.t. Genus Frankliniella Karny, 1910

1. Frankliniella schultzei (Trybom, 1910)

III.D. u. Genus Kurtomathrips Moulton, 1927

1. Kurtomathrips morrilli Moulton, 1927

III.D.v. Genus Megalurothrips Bagnall, 1915

1. Megalurothrips peculiaris (Bagnall, 1918)

2. Megalurothrips usitatus (Bagnall, 1913)

III.D.w. Genus Microcephalothrips Bagnall, 1926

1. Microcephalothrips abdominalis (Crawford, 1910)

III.D.x. Genus Mycterothrips Trybom, 1970

1. Mycterothrips ricini (Shumsher, 1946)

III.D.y. Genus Organothrips Hood, 1940

1. Organothrips indicus Bhatti, 1974

III.D.z. Genus Parexothrips Priesner, 1965

1. Parexothrips tenellus (Priesner, 1950)

III.D.aa. Genus Plutonothrips Priesner, 1933

1. Plutonothrips cus (Bhatti, 1967)

III.D.ab. Genus Priesneriola Ananthakrishnan, 1964

1. Priesneriola oneillae Ananthakrishnan, 1964

III.D.ac. Genus Psilothrips Hood, 1927

1. Psilothrips indicus Bhatti, 1967

III.D.ad. Genus Rhamphothrips Karny, 1913

1. Rhamphothrips jasminae (Bhatti, 1977)

2. Rhamphothrips parviceps (Hood, 1919)

III.D.ae. Genus Salpingothrips Hood, 1935

1. Salpingothrips hoodi Ananthakrishnan, 1969

III.D.af. Genus Scirtothrips Shull, 1909

1. Scirtothrips dorsalis Hood, 1919
2. Scirtothrips oligochaetus (Karny, 1927)

3. Scirtothrips mangiferae Priesner, 1932

III.D.ag. Genus Scolothrips Hinds, 1902

1. Scolothrips asura Ram. \& Marg., 1931

2. Scolothrips rhagebianus Priesner, 1950

3. Scolothrips tenuipennis zur Strassen, 1965

III.D.ah. Genus Sorghothrips Priesner, 1936

1. Sorghothrips fuscus Ananthakrishnan, 1965

2. Sorghothrips jonnaphilus (Ramakrishna, 1928)

III.D.ai. Genus Stenchaetothrips Bagnall, 1926

1. Stenchaetothrips biformis (Bagnall, 1913)

2. Stenchaetothrips faurei (Bhatti, 1962)

3. Stenchaetothrips indicus (Ram. \& Marg., 1931)

III.D.aj. Genus Tenothrips Bhatti, 1967

1. Tenothrips frici (Uzel, 1895)

III.D.ak. Genus Thrips Linnaeus, 1758

1. Thrips apicatus Priesner, 1934

2. Thrips carthami Shumsher, 1946

3. Thrips chandni Bhatti, 1999

4. Thrips coloratus Schmutz, 1913

5. Thrips flavus Schrank, 1776

6. Thrips florum Schmutz, 1913

7. Thrips garuda Bhatti, 1980)

8. Thrips hawaiiensis (Morgan, 1913)

9. Thrips orientalis (Bagnall, 1915)

10. Thrips palmi Karny, 1925

11. Thrips speratus zur Strassen, 1978

12. Thrips subnudula (Karny, 1927)

13. Thrips tabaci Lindeman, 1889

III.D.al. Genus Tusothrips Bhatti, 1967

1. Tusothrips sumatrensis (Karny, 1925)

\section{Synonymy and Comments}

I. Family: Aeolothripidae Uzel, 1895

1895. Aeolothripidae Uzel, Monogr. Ord. Thysanopt., pp. $27,42,62$.

In India there are 15 species in 9 genera (Bhatti, 1990: 207). Aduncothrips Ananthakrishnan, 1966 (1 species), Allelothrips Bagnall, 1932 (1 species), Coleothrips Haliday, 1836 (6 species), Franklinothrips Back, 1912 (1 species), Gelothrips Bhatti, 1967 (1 species), Indothrips Bhatti, 1967 (1 species), Mymarothrips Bagnall, 1928 (2 species), Orothrips Moulton, 1907 (1 species), Streothrips Bhatti, 1971 (1 species). 3 species in 2 genera are found in Delhi, Coleothrips collaris (Priesner, 1919), Coleothrips mongolicus (Pelikán, 1985), and Indothrips bhushani Bhatti, 1967.

I. a. Genus Coleothrips Haliday

1836. Aeolothrips subgenus Coleothrips Haliday, Ent. Mag., 3 (5): 451.

1988. Coleothrips Bhatti, Zoology, 1(2): 115-116.

1. Coleothrips collaris (Priesner)

1919. Aeolothrips fasciatus var. collaris Priesner, Sitz.-ber. Akad. Wiss. Wien, (1), 128(2-3): 119120.

1988. Coleothrips collaris Bhatti, Zoology, 1(2): 115.

2. Coleothrips mongolicus (Pelikán)

1985. Aeolothrips mongolicus Pelikán, Ann. Hist.-

Nat. Mus. Natn. Hungarici, 77: 128-129.

1988. Coleothrips mongolicus Bhatti, Zoology, 1(2): 116

I. b. Genus Indothrips Bhatti

1967. Indothrips Bhatti, Thysanoptera Nova Indica, p. 2.

1. Indothrips bhushani Bhatti

1967. Indothrips bhushani Bhatti, Thysanoptera Nova Indica, p. 2-3.

II Family: Stenurothripidae Bagnall

1923. Stenurothripidae Bagnall, Ent. Mon. Mag., (3) 
9: 37 .

The family Stenurothripidae includes 2 species in 1 genus from India. (Bhatti 1990: 207): Holarthrothrips indicus Bhatti \& Ananthakrishnan 1978, H. jambudvipae (Ramakrishna 1928). Only Holarthrothrips indicus is found in Delhi.

II.a. Genus Holarthrothrips Bagnall

1927. Holarthrothrips Bagnall, Ann. Mag. Nat. Hist., (9)20: 562-563.

1. Holarthrothrips indicus Bhatti \& Anan.

1978. Holarthrothrips indicus Bhatti \&

Ananthakrishnan, Entomon, 3(2): 229-230.

\section{Family: Thripidae Stephens 1829}

1829. Thripidae Stephens, Syst. Cat. Brit. Ins., p. 363.

Family Thripidae includes 267 species in 111 genera from India (Bhatti 1990: 208). Eighty-two species in 48 genera are found in Delhi within 4 subfamilies (Bhatti 1990: 208): Dendrothripinae, Sericothripinae, Panchaetothripinae, and Thripinae.

III.A. Subfamily: Dendrothripinae Priesner 1925 1925. Dendrothripini Priesner, Konowia, 4(3-4): 144. 1948.

Dendrothripinae Morison, London Natur. Suppl., 59(2): 41, 50.

In India, 5 genera with 19 species are found (Bhatti 1990: 208). In Delhi, 2 genera with 4 species are found: Dendrothrips (2 species), Pseudodendrothrips (2 species).

\section{III.A.a.Genus Dendrothrips Uzel}

1895. Dendrothrips Uzel, Monogr. Ord. Thysanopt., p. 159.

1. Dendrothrips cameroni Priesner

1965. Dendrothrips cameroni Priesner, Publ. Inst. Desert Egypte (1960), 13: 282. Sudan.

2. Dendrothrips punctatus zur Strassen

1968. Dendrothrips punctatus zur Strassen, Senckenbergiana biol., 49(1): 28.

III.A.b. Genus Pseudodendrothrips Schmutz 1913 1913. Pseudodendrothrips Schmutz, Sitz.-ber. Akad. Wiss. Wein, math.-naturw. KI., Abt. I, 122(7): 998999

1. Pseudodendrothrips albana Bhatti

1997. Pseudodendrothrips albana Bhatti, Zool. Surv. India State Fauna Series 6, Fauna of Delhi, pp. 296-297.

2. Pseudodendrothrips suvarna Bhatti 1997

1997. Pseudodendrothrips suvarna Bhatti, Zool. Surv. India State Fauna Series 6, Fauna of Delhi, pp. 297-299.

III.B. Subfamily: Panchaetothripinae Bagnall 1912 1912. Panchaetohripinae Bagnall, Rec. Indian Mus., 7(3): 258.

In India 18 genera with 31 species are found (Bhatti 1990: 208). In Delhi this subfamily comprises 5 genera with 7 species: Astrothrips (1 species), Brevithrips (1 species), Caliothrips (3 species), Phibalothrips (1 species), and Rhipiphorothrips (1 species).

III.B.a. Genus Astrothrips Karny

1921. Astrothrips Karny, Treubia, 1(4): 215, 239.

1. Astrothrips stannardi Bhatti

1967. Astrothrips stannardi Bhatti, Thysanoptera Nova Indica, pp. 7-8.

III.B.b. Genus Brevithrips Bhatti

1967. Brevithrips Bhatti, Thysanoptera Nova Indica, p. 8 .

1. Brevithrips tumiceps (Karny)

1923. Astrothrips tumiceps Karny, Treubia, 3(3-4): 331-334.

1967. Brevithrips tumiceps Bhatti, Thysanoptera Nova Indica, p. 8 .
III.B.c. Genus Caliothrips Daniel

1904. Caliothrips Daniel, Ent. News, 15(9): 296.

1. Caliothrips graminicola (Bag. \& Cam.)

1932. Hercothrips graminicola Bagnall and Cameron, Ann. Mag. Nat. Hist., 10(10): 417-419.

1957. Caliothrips graminicola Faure, Jour. Entomol. Soc. S. Africa, 20(1): 79-88.

2. Caliothrips indicus (Bagnall)

1913. Heliothrips indicus Bagnall, Ann. Mag. Nat. Hist., (8)12: 291.

1956. Caliothrips indicus Ananthakrishnan, A survey of our present knowledge of Indian Thysanoptera, p. 3. Amar Press: Madras.

3. Caliothrips luckmanni Wilson

1975. Caliothrips luckmanni Wilson, Mem. Amer. Ent. Inst., 23: 72 (in key to species), 86- 88.

III.B.d. Genus Phibalothrips Hood

1918. Phibalothrips Hood, Mem. Queensland Mus., 6 125.

1. Phibalothrips peringueyi (Faure)

1925. Reticulothrips peringueyi Faure, S. Afr. J. Nat. Hist., 5: 145-150.

1937. Phibalothrips peringueyi Jacot-Guillarmodi,

Publ. Univ. Pretoria, (II) 3: 10

III.B.e. Genus Rhipiphorothrips Morgan

1913. Rhipiphorothrips Morgan, Proc. U. S. Nat. Mus., 46(2008): 17.

1. Rhipiphorothrips cruentatus Hood

1919. Rhipiphorothrips cruentatus Hood, Insec. Inscit. Menstr., 7(4-6): 94-96.

III.C. Subfamily: Sericothripinae Karny 1921

1921. Sericothripinae Karny, Treubia, 1(4): 236, 237.

Eleven species in six genera are found in India (Bhatti 1990: 208): Corcithrips Bhatti 1973 (1 species), Elbuthrips (1 species), Hydatothrips Karny 1913 (6 species), Kazinothrips Bhatti 1973 (1 species), Neohydatothrips John 1929 (1 species), Pyrothrips Bhatti 1973 (1 species).

Six species in three genera are found in Delhi: Hydatothrips Karny 1913 (2 species), Neohydatothrips John 1929 (3 species), Pyrothrips Bhatti 1973 (1 species).

III.C.a. Genus Hydatothrips Karny

1913. Hydatothrips Karny, Wiss. Ergeb. Zentral-Africa-

Exped., 1907-1908, Zoologie, 4(10): 281

1. Hydatothrips atactus Bhatti

1973. Hydatothrips atactus Bhatti, Oriental Ins. 7(3): 418-432.

2. Hydatothrips ramaswamiahi Priesner

1926. Sericothrips ramaswamiahi Priesner, Treubia, 8: 51 .

1973. Hydatothrips ramaswamiahi Bhatti, Oriental Ins. 427-432.

III.C.b. Genus Neohydatothrips John

1929. Neohydatothrips John, Bull. et Ann. Soc. Ent. Belgique, 69: 33-34.

1. Neohydatothrips gracilipes (Hood)

1924. Sericothrips gracilipes Hood, Canadian Ent., 56(6): 149-150.

1987. Neohydatothrips gracilipes Sakimura, Plant Disease, 71: 507.

2. Neohydatothrips raniae (Bhatti)

1967. Sericothrips raniae Bhatti, Thysanoptera Nova Indica, pp. 9-10.

1973. Neohydatothrips raniae Bhatti, Oriental Ins., 7(3): 435 .

3. Neohydatothrips samayunkur (Kudô)

1995. Hydatothrips (Neohydatothrips) samayunkur

Kudô, Appl. Ent. Zool., 30: 169-176.

1999. Neohydatothrips samayunkur Nakahara, Proc.

Ent. Soc. Wash., 101(2): 458-459.

III.C.c. Genus Pyrothrips Bhatti

1973. Hydatothrips subgenus Pyrothrips Bhatti, Oriental Ins., 7(3): 424

1990. Pyrothrips Bhatti, Zoology, 2(4): 247.
1. Pyrothrips boerhaaviae (Sesh. \& Anan.)

1954. Sericothrips (Hydatothrips) boerhaaviae Seshadri \& Ananthakrishnan, Indian J. Ent., 16(3): 210-212.

1990. Pyrothrips boerhaaviae Bhatti, Zoology, 2(4): 247.

III.D. Subfamily: Thripinae Karny 1921

1921. Thripinae Karny, Treubia, 1(4): 215, 236, 239.

This subfamily includes most of the genera of family Thripidae, and is most difficult to define. It can be recognized by the absence of characters, which distinguish the other subfamilies. In India, there are 206 species in 86 genera (Bhatti 1990: 208). In Delhi, 38 genera with 65 species are found.

III.D.a. Genus Agrostothrips Hood

1954. Agrostothrips Hood, Rev. Zool. Bot. Afr., 49(12): 1 .

1. Agrostothrips meridionalis (Bagnall)

1927. Chirothrips meridionalis Bagnall, Ann. Mag Nat. Hist., (9)19: 566

1990. Agrostothrips meridionalis Bhatti, Zoology, 2(4): 194.

III.D.b. Genus Ajothrips Bhatti

1967. Ajothrips Bhatti, Bull. Ent., 8(1): 58.

1. Ajothrips medius Bhatti

1997. Ajothrips medius Bhatti, Zool. Surv. India State

Fauna Series 6, Fauna of Delhi, pp. 301-302.

III.D.c. Genus Alathrips Bhatti

1969. Alathrips Bhatti, Oriental Ins., 3(4): 373.

1. Alathrips roonwali (Bhatti)

1963. Hyalopterothrips roonwali Bhatti, J. Zool. Soc. India (1962), 14(2): 177-178.

1969. Alathrips roonwali Bhatti, Oriental Ins., 3(4): 373.

III.D.d. Genus Anaphothrips Uzel

1895. Anaphothrips Uzel, Monogr. Ord. Thysanopt. pp. 142-143.

1. Anaphothrips sudanensis Trybom

1911. Anaphothrips sudanensis Trybom, Res. Swed.

Zool. Exped. Egypt \& White Nile 1901, 4(19): 6063.

III.D.e. Genus Anascirtothrips Bhatti

1961. Anascirtothrips Bhatti, Bull. Ent., 2: 26.

1. Anascirtothrips arorai Bhatti

1961. Anascirtothrips arorai Bhatti, Bull. Ent., 2: 26-28.

III.D.f. Genus Ayyaria Karny

1927. Ayyaria Karny, Mem. Dept. Agric. India, Ent. Ser. (1926), 9(6): 193

1. Ayyaria chaetophora Karny

1927. Ayyaria chaetophora Karny, Mem. Dept. Agric. India, Ent. Ser. (1926), 9(6): 193-195.

III.D.g. Genus Bathrips Bhatt

1962. Bathrips Bhatti, Bull. Ent., 3: 34

1. Bathrips melanicornis (Shumsher)

1946. Taeniothrips melanicornis Shumsher, Indian J. Ent., 7(1-2): 161 (in key), 179-181.

1962. Bathrips melanicornis Bhatti, Bull. Ent., 3:3435.

III.D.h. Genus Bolacothrips Uzel

1895.Bolacothrips Uzel, Monogr. Ord. Thysanopt., pp. 211-212.

1. Bolacothrips striatopennatus (Schmutz)

1913. Thrips striatopennata Schmutz, Sitz.-Ber. Akad. Wiss. Wien, math.-naturw. KI. Abt. I, 122(7): 1002-1003.

1983. Bolacothrips striatopennatus Bhatti, Ann. Nathist. Mus. Wien, 84: 490-500.

III.D.i. Genus Bregmatothrips Hood

1912. Bregmatothrips Hood, Proc. Biol. Soc.

Washington, 25: 66-67.

1. Bregmatothrips brachycephalus (Shumsher) 
1942. Neolimothrips brachycephalus Shumsher, Indian J. Ent., 4(2): 118-120.

1968. Bregmatothrips brachycephalus Mound, Bull. Brit. Mus. (Nat. Hist.) Ent. Suppl., 11: 28-29.

III.D.j. Genus Ceratothripoides Bagnall

1918. Ceratothripoides Bagnall, Ann. Mag. Nat. Hist., (9) 1: 201.

1. Ceratothripoides claratris (Shumsher)

1946. Taeniothrips claratris Shumsher, Indian J. Ent. (1945), 7(1-2): 178-179.

1990. Ceratothripoides claratris Bhatti, Zoology, 2(4): 219

III.D.k. Genus Chirothrips Haliday

1836. Thrips (Chirothrips) Haliday, Ent.. Mag., 3: 444.

1843. Chirothrips Amyot \& Serville 1843, Hist. Nat. Ins. Hémipt., p. 642

1. Chirothrips africanus Priesner

1932. Chirothrips africanus Priesner, Bull. Soc. Ent. Egypte, 16: 46-47.

III.D.I. Genus Craspedothrips zur Strassen

1966. Craspedothrips zur Strassen, Senckenbergiana Biol., 47(6): 444-445.

1. Craspedothrips minor (Bagnall)

1921. Physothrips minor Bagnall, Ann. Mag. Nat. Hist., (9)8: 393-394.

1968. Craspedothrips minor Mound, Bull. Brit. Mus.

(Nat. Hist.) Ent. Suppl., 11: 32-33.

III.D.m. Genus Dendrothripoides Bagnall

1923. Dendrothripoides Bagnall, Ann. Mag. Nat. Hist., (9) $12: 624$.

1. Dendrothripoides innoxius (Karny)

1914. Euthrips innoxius Karny, Zeitschr. Wiss. Ins.biol., 10(10-12): 359-360.

1978. Dendrothripoides innoxius Bhatti, Oriental Ins., 12(1): 10 .

III.D.n. Genus Diarthrothrips Williams

1915. Diarthrothrips Williams, Bull. Ent. Res., 6: 269.

1. Diarthrothrips nimbus (Ananthakrishnan)

1965. Mycterothrips nimbus Ananthakrishnan, Bull.

Ent., 6: 20.

1978. Diarthrothrips nimbus Bhatti \&

Ananthakrishnan, Entomon, 3 (2) : 231.

III.D.o. Genus Dichromothrips Priesner

1932. Dichromothrips Priesner, Stylops, 1(5): 110.

1. Dichromothrips corbetti (Priesner)

1936. Dichromothrips corbetti Priesner, Proc. Roy.

Ent. Soc. London, Ser B, 5(11): 209-210.

2. Dichromothrips indicus Mound

1976. Dichromothrips indicus Mound, Biol. J. Linnean

Soc., 8(3); 255-256.

III.D.p. Genus Eremiothrips Priesner

1949. Eremiothrips Priesner, Bull. Soc. Fouad Ent., 33: 46 (differentiating characters given in key to genera), 129 (type species designated).

1. Eremiothrips varius (Bhatti)

1967. Ascirtothrips varius Bhatti, Thysanoptera Nova Indica, pp. 13-14.

1988. Eremiothrips varius Bhatti, Zoology, 1(2): 123.

III.D.q. Genus Exothrips Priesner

1939. Exothrips Priesner, Rev. Zool. Bot. Afr., 32(2):

162.

1. Exothrips cephalicus Bhatti

1975. Exothrips cephalicus Bhatti, Oriental Ins., 9(1):

57-58.

2. Exothrips deemax Bhatti

1975. Exothrips deemax Bhatti, Oriental Ins., 9(1): 58-59.

3. Exothrips hemavarna (Ram. \& Marg.)

1931. Oxythrips hemavarna Ramakrishna \& Margabandhu, J. Bombay Nat. Hist. Soc., 34 (4): 1036.

1969. Exothrips hemavarna Sakimura \&

Ananthakrishnan, Bull. Ent., 3: 53-56.

4. Exothrips ornus Bhatti
1975. Exothrips ornus Bhatti, Oriental Ins., 9(1): 60-61.

5. Exothrips poorva Bhatti

1975. Exothrips poorva Bhatti, Oriental Ins., 9(1): 61-62.

6. Exothrips redox Bhatti

1975. Exothrips redox Bhatti, Oriental Ins., 9(1): 62-64.

III.D.r. Genus Florithrips Bhatt

1970. Florithrips Bhatti, Oriental Ins. (1969), 3(4): 377.

1. Florithrips traegardhi (Trybom)

1911. Physapus traegardhi Trybom, Res. Swed

Zool. Exped. Egypt and White Nile 1901, 4: 4-6.

1970. Florithrips traegardhi Bhatti, oriental Ins.

(1969), 3(4): 377

III.D.s. Genus Foliothrips Bhatti

1972. Foliothrips Bhatti, Oriental Ins., 6(4): 543.

1. Foliothrips oratus Bhatti

1972. Foliothrips oratus Bhatti, Oriental Ins., 6(4): 543-544.

III.D.t. Genus Frankliniella Karny

1910. Frankliniella Karny, Mitt. Naturw. Ver. Univ.

Wein, 8(2): 46.

1. Frankliniella schultzei (Trybom)

1910. Physopus schultzei Trybom, Denks. Med.-

natur. Sch. Gesell. XVI, 4(1): 151-153.

1948. Frankliniella schultzei Moulton, Revista de

Entomologica, 19 () 1-2: 78, 100.

III.D.u. Genus Kurtomathrips Moulton

1927. Kurtomathrips Moulton, Bull. Brooklyn Ent. Soc.

22(4): 187-188.

1. Kurtomathrips morrilli Moulton

1927. Kurtomathrips morrilli Moulton, Bull. Brooklyn.

Ent. Soc., 22(4): 188-190.

III.D.v. Genus Megalurothrips Bagnall

1915. Megalurothrips Bagnall, Ann. Mag. Nat. Hist.,

(8)15: 589.

1. Megalurothrips peculiaris (Bagnall)

1918. Physothrips peculiaris Bagnall, Ann. Mag.

Nat. Hist., (9)1(3): 206-207.

1969. Megalurothrips peculiaris Bhatti, Oriental Ins., 3(3): 242.

2. Megalurothrips usitatus (Bagnall)

1913. Physothrips usitatus Bagnall, Ann. Mag. Nat. Hist., (8)12(69): 293-294.

1972. Megalurothrips usitatus Sakimura, Kontyû, 40(3): 192.

III.D.w. Genus Microcephalothrips Bagnall

1926. Microcephalothrips Bagnall, Ann. Mag. Nat.

Hist., (9)18: 113.

1. Microcephalothrips abdominalis (Crawford)

1910. Thrips abdominalis D. L. Crawford, Pomona

Coll. Jour. Ent., 2(1): 157-159.

1926. Microcephalothrips abdominalis Bagnall, Ann.

Mag. Nat. Hist., (9)18: 113-114.

III.D.x. Genus Mycterothrips Trybom

1910. Mycterothrips Trybom, Denkschr. Med.Naturwiss. Ges., 4(1): 158.

1. Mycterothrips ricini (Shumsher)

1946. Taeniothrips (Rhoplandrothrips) ricini Shumsher, Indian J. Ent. (1945), 7(1-2): 176-178.

1970. Mycterothrips ricini Bhatti, Oriental Ins., 3(4): 378.

III.D.y. Genus Organothrips Hood

1940. Organothrips Hood, Proc. Hawaiian Ent. Soc., 10(3): 423.

1. Organothrips indicus Bhatti

1974. Organothrips indicus Bhatti, Oriental Ins., 8(2): 149-153.

III.D.z. Genus Parexothrips Priesner

1965. Parexothrips Priesner, Publ. Inst. Desert

Egypte (1960), 13: 285-286.

1. Parexothrips tenellus (Priesner 1950)
1950. Exothrips tenellus Priesner, Bull. Soc. Faud. Ent., 34: 30-32.

1965. Parexothrips tenellus Priesner, Publ. Inst. Desert Egypte (1960), 13: 286-288.

III.D.aa. Genus Plutonothrips Priesner

1933. Plutonothrips Priesner, Konowia, 12(3-4): 307

1. Plutonothrips cus (Bhatti)

1967. Arathrips cus Bhatti Thysanoptera Nova Indica, pp. 16

1984. Plutonothrips cus Bhatti, Ann, Entomol., 2(1): 92.

III.D.ab. Genus Priesneriola Ananthakrishnan

1964. Priesneriola Ananthakrishnan, Bull. Ent., 5: 52.

1. Priesneriola oneillae Ananthakrishnan

1964. Priesneriola oneillae Ananthakrishnan, Bull. Ent., 5: 53-54.

III.D.ac. Genus Psilothrips Hood

1927. Psilothrips Hood, Proc. Biol. Soc. Washington, 40: 198

1. Psilothrips indicus Bhatti

1967. Psilothrips indicus Bhatti, Thysanoptera Nova Indica, p. 12.

III.D.ad. Genus Rhamphothrips Karny

1912. Rhynchothrips Karny, Zool. Anz., 40(10-11): 297.

1978. Rhamphothrips Bhatti, Oriental Ins., 12(3): 282-285 .

1. Rhamphothrips jasminae (Bhatti)

1977. Perissothrips jasminae Bhatti, Oriental Ins., 11(4): 572-573.

1978. Rhamphothrips jasminae Bhatti, Oriental Ins., 12(3): 289.

2. Rhamphothrips parviceps (Hood)

1919. Perissothrips parviceps Hood, Insect. Inscit Menstr., 7(4-6): 92-93.

1978. Rhamphothrips jasminae Bhatti, Oriental Ins., 12(3): 291-293.

III.D.ae. Genus Salpingothrips Hood

1935. Salpingothrips Hood, J. New York Ent. Soc., 43: 157.

1. Salpingothrips hoodi Ananthakrishnan

1969. Salpingothrips hoodi Ananthakrishnan, Oriental Ins., 2(2):202-204.

III.D.af. Genus Scirtothrips Shull

1909. Scirtothrips Shull, Ent. News, 20(5): 222.

1. Scirtothrips dorsalis Hood

1919. Scirtothrips dorsalis Hood, Insec. Inscit. Menstr., 7(4-6): 90-91.

2. Scirtothrips mangiferae Priesner

1932. Scirtothrips mangiferae Priesner, Bull. Soc. Ent. Egypte, 16: 143-145.

3. Scirtothrips oligochaetus (Karny)

1927. Anaphothrips oligochaetus Karny, Mem. Dept. Agric. India Ent. Ser., 9(6): 201-203.

III.D.ag. Genus Scolothrips Hinds

1902. Scolothrips Hinds, Proc. U. S. Natnl. Mus. 26(1310): 157

1. Scolothrips asura Ram. \& Marg.

1931. Scolothrips asura Ramakrishnan \&

Margabandhu, J. Bombay Nat. Hist. Soc., 34(4): 1035-1036.

2. Scolothrips rhagebianus Priesner

1950. Scolothrips rhagebianus Priesner, Bull. Soc.

Fouad ler Ent., 34: 46-48.

3. Scolothrips tenuipennis zur Strassen

1965. Scolothrips tenuipennis zur Strassen, Comm. Biol. Soc. Scient. Fennica, 28(6): 30-32.

III.D.ah. Genus Sorghothrips Priesner

1936. Sorghothrips Priesner, Bull. Soc. Roy. Ent. Egypte, 20: 88

1. Sorghothrips fuscus Ananthakrishnan

1965. Ornothrips fuscus Ananthakrishnan, Bull. Ent., 6: 17-18. 
1969. Pellothrips fuscus Ananthakrishnan, Oriental Ins. (1968), 2(2): 204.

1969. Sorghothrips fuscus Bhatti, Oriental Ins., 3(4): 380.

2. Sorghothrips jonnaphilus (Ramakrishna)

1928. Taeniothrips jonnaphila Ramakrishna, Mem. Dept. Agric. India Ent. Ser., 10(7): 256-258.

1942. Ramakrishnothrips jonnaphila Shumsher, Indian J. Ent., 4(2): 117.

1969. Sorghothrips jonnaphilus Bhatti, Oriental Ins. 3(4): 380 .

III.D.ai. Genus Stenchaetothrips Bagnall

1926. Stenchaetothrips Bagnall, Ann. Mag. Nat. Hist., (9)18: 107.

1980. Stenchaetothrips Bhatti and Mound, Bull. Ent.,

21(1-2): 13

1. Stenchaetothrips biformis (Bagnall)

1913. Bagnallia biformis Bagnall, J. Econ. Biol., 8(4): 237-238.

1980. Stenchaetothrips biformis Bhatti \& Mound, Bull. Ent., 21(1-2): 14-15.

2. Stenchaetothrips faurei (Bhatti)

1962. Chloethrips faurei Bhatti, Bull. Ent., 3: 43-45.

1980. Stenchaetothrips faurei Bhatti \& Mound, Bull. Ent., 21(1-2): 15.

3. Stenchaetothrips indicus (Ram. \& Marg.)

1931. Fulmekiola indica Ramakrishna \& Margabandhu, J. Bombay Nat. Hist. Soc., 34(4): 1034-1035

1980. Stenchaetothrips indicus Bhatti \& Mound, Bull. Ent., 21: 15.

III.D.aj. Genus Tenothrips Bhatti

1967. Taeniothrips subgenus Tenothrips Bhatti, Thysanoptera Nova Indica, p. 18.

1990. Tenothrips Bhatti, Zoology, 2(4): 201

1. Tenothrips frici (Uzel)

1895. Physopus friciUzel, Monogr. Ord. Thysanopt. pp. 126-127.

1920. Taeniothrips frici Priesner, Jahresber. Oberösterr. Mus.-Ver. Franc. Carol., 78: 54. 1990. Tenothrips frici Bhatti, Zoology, 2 (4): 203.

III.D.ak. Genus Thrips Linnaeus

1758. Thrips Linnaeus, Syst. Nat., 10th ed., p. 457.

1. Thrips apicatus Priesner

1934. Thrips apicatus Priesner, Natuurk. Tijdschr.

Ned.-Indie, 94(3): 264, 286 (in key)

2. Thrips carthami Shumsher

1946. Thrips carthami Shumsher, Indian, J. Ent. (1945), 7(1-2): 184-185

3. Thrips chandni Bhatti

1999. Thrips chandni Bhatti, Thrips, 1: 58-61, figs. 1-5.

4. Thrips coloratus Schmutz

1913. Thrips coloratus Schmutz, Sitz.-ber. Akad. Wiss. Wein, math.-naturw. KI., Abt. I, 122(7): 10131015.

5. Thrips flavus Schrank

1776. Thrips flavus Schrank, Beitr. Naturg., pp. 3133.

6. Thrips florum Schmutz 1913

1913. Thrips florum Schmutz, Sitz.-ber. Akad. Wiss. Wein, math.-naturw. KI., Abt. I, 122(7): 1003-1005.

7. Thrips garuda Bhatti

1980. Thrips garuda Bhatti, Syst. Ent., 5: 137.

8. Thrips hawaiiensis (Morgan)

1913. Euthrips hawaiiensis Morgan, Proc. U. S. Nat. Mus., 46: 3-5.

1934. Thrips hawaiiensis Priesner, Natuurk. Tijdschr. Ned.-Ind., 94(3): 266-267, 287 (in key).

9. Thrips orientalis (Bagnall)

1915. Isoneurothrips orientalis Bagnall, Ann. Mag. Nat. Hist., (8)15: 593-594.

1978. Thrips orientalis Bhatti, Oriental Ins., 12(2): 191.

10. Thrips palmi Karny

1925. Thrips palmi Karny, Bull. deli Proefst. Medan, 23: $10-15$.

11. Thrips subnudula (Karny)
1927. Ramaswamiahiella subnudula Karny, Mem. Dept. Agric. India, Ent. Ser., 9(6): 208-210.

2005. Thrips subnudula Mound \& Masumoto, Zootaxa, 1020: 50.

12. Thrips speratus zur Strassen

1978. Thrips speratus zur Strassen,

Senckenbergiana Biol., 59(3-4): 241-245.

13. Thrips tabaci Lindeman

1889. Thrips tabaci Lindeman, Bull. Soc. Imp. Nat. Moscou, 1: 61-75.

\section{REFERENCES}

Ananthakrishnan T.N. \& S. Sen (1980). Taxonomy of Indian Thysanoptera. Z.S.I. Handbook Series No. 1: 234pp.

Bailey, S F. (1957). The thrips of California part 1: Suborder: Terebrantia. Bulletin of the California Insect Survey 4: 143-220.

Bhatti, J.S. (1964). Studies on the Indian species of the genus Aeolothrips Hal. Bulletin of Entomology 5: $17-23$.

Bhatti, J.S. (1990). Catalogue of Insects of the Order Terebrantia from the Indian subregion. Zoology 2(4): 205-352

Bhatti, J.S. (1997). Thysanoptera, pp.291-324. In: Fauna of Delhi. Zoological Survey of India, State Fauna Series 6

Bhatti, J.S. (1999). Yellow dorsally spotted species of Thrips (Terebrantia: Thripidae) in India with description of a new species in flowers of Tabernaemontana (Apocynaceae) and Lantana (Verbenaceae). Thrips 1: 58-65.

Bhatti, J.S., V. Veer \& A. Dahiya (1999). The North American species Kurtomathrips morrilli Moulton and Neohydatothrips gracilipes (Hood) (Terebrantia: Thripidae) have now established large populations from North to South India. Thrips 1: 54-57. Delhi.

Bhatti, J.S., R. Varatharajan, S.C. Vareichung, V. Kumar \& K. Tyagi (2003). The exotic species Neohydatothrips samayunkur (Kudô, 1995) (Terebrantia: Sericothripidae) is now discovered from 6 states in India, pp.666-667. In: Proceedings of the National Symposium. Subrahmanyam, B. \& V.V. Ramamurthy (eds), Frontier Areas of Entomological Research 5-7 Nov. 2003, Delhi

Hinds, W.F. (1902). Contribution to a monograph of the insects of order Thysanoptera inhabiting North America. Proceedings of the U. S. National Museum 26(1310): 79-272.

Kumar, V., K. Tyagi \& J.S. Bhatti (2005). On some new records of Thysanoptera from India. Entomon 30(3): 294-254.

Mound, L.A. (1976). Thysanoptera of the genus Dichromothrips of the Old World Orchidaceae. Biological Journal of Linnean Society London 8(3): 245-265.

Mound, L.A. (2002). So many thrips - a few tospoviruses?, pp.15-18. In: Thrips and Tospoviruses: Proceedings of the 7th International Symposium on Thysanoptera. Marullo, R. \& L.A. Mound (eds.). Published as CD-ROM file by Australian National Insect Collection, Canberra. (December), 390 pp.

Mound, L.A. \& T. Marullo (1996). The Thrips of Central and South America: An Introduction (Insecta: Thysanoptera). Memoirs on Entomology International 6: 1-487. Associate Publishers: Gainesville (Florida).

Mound, L.A., G.D. Morison, B.R. Pitkin \& J.M. Palmer (1976). Thysanoptera. Royal Entomological Society of London 1(11): 1-79.

Mound, L.A. \& A.K. Walker (1982). Terebrantia (Insecta: Thysanoptera). Fauna of New Zealand 1: $1-113$.

Priesner, H. (1926-1928). Die Thysanopteren Europas. Teil I-IV. 755pp. Wein. (In German).

Priesner, H. (1964). Ordnung Thysanoptera (Fransenflügler, Thripse). Bestimmungsbücher zur Bodenfauna Europas, Lief. 2, p. 1-242. Berlin. (In German).

Priesner, H. (1965). A monograph of the Thysanoptera of the Egyptian deserts. Publications de l'Institut du Désert d'Egypte (1960), No. 13: 549 pp. Cairo.

Schliephake, G. (1979). pp.1-332. In: Schliephake, G. \& K. Klimt (1979). Thysanoptera, Frasenflügler. Die Tierwelt Deutschlands, 66: 477 pp. Gustav Fischer: Jena (In German).

Ramakrishna Ayyar, T.V. \& V. Margabandhu (1940). Catalogue of Indian Insects. pt. 25, Thysanoptera. Pp. iv +64 . New Delhi.

Singh, S. (1942). A contribution to our knowledge of Indian Thysanoptera. Indian Journal of Entomology 4(2): 111-135. New Delhi.

Stannard, L.J. (1968). The Thrips, or Thysanoptera, of Illinois. Illinois Natural History Survey Bulletin 29(4): 215-552.

Uzel, H. (1895). Monographie der Ordnung Thysanoptera, 472 pp., 10 pls. Published by the author: Königgratz, Bohemia. (In Bohemian \& German).

\section{ACKNOWLEdGement}

We are grateful to Dr. V.V. Ramamurthy, Principal Scientist (Division of Entomology, IARI, New Delhi) for his moral support, suggestions and introducing us to Zoos' Print Journal. 